\title{
Hypoglyceamia in a Patient with a Solitary Fibrous Tumour
}

\author{
Andrew Okpe, Kerri Ramsay, Isuru P Fernando, Emily Mudenha, Devaka JS Fernando \\ Department of Diabetes and Endocrinology, King's Mill Hospital, Sutton-in-Ashfield, Nottinghamshire, UK
}

Doi: 10.12890/2016_000353- European Journal of Case Reports in Internal Medicine - C EFIM 2016

Received: 05/12/2015

Accepted: 31/01/2016

Published: 07/03/2016

How to cite this article: Okpe A, Ramsay K, Fernando IP, Mudenha E, Fernando DJS. Hypoglyceamia in a patient with a solitary fibrous tumour. EJCRIM 2016;3:doi:10.12890/2016_000353

Conflicts of Interests: The authors declare that there are no competing interests.

This article is licensed under a Commons Attribution Non-Commercial 4.0 License

\section{ABSTRACT}

Objective: To illustrate an unusual mechanism causing hypoglycaemia.

Material and methods: A 76-year-old man presented with episodes of agitation and confusion and was resuscitated with oral glucose gel when found to be hypoglycaemic.

Results: A CT scan for an abdominal mass confirmed a solitary fibrous tumour (SFT). The sarcoma multidisciplinary team suggested conservative management. The patient's episodic hypoglycaemia was managed with diet modification including corn-based starch, scheduled snacks and dexamethasone. Glucose levels were within normal range at discharge from hospital. The patient was referred to the palliative care team for follow-up.

Conclusion: SFTs causing non-islet cell tumour hypoglycaemia are difficult to treat.

\section{LEARNING POINTS}

- To be aware of rarer causes of hypoglycaemia and to consider unusual causes in acute presentations of hypoglycaemia, especially in patients who do not have diabetes.

- This case illustrates the importance of a thorough general physical and systemic examination, as identifying the abdominal mass is essential to the early diagnosis of this rare condition.

- Solitary fibrous tumours causing non-islet cell tumour hypoglycaemia are difficult to treat, and even when surgical resections are applicable, recurrence rates are high.

\section{KEYWORDS}

Hypoglycaemia; non insulin mediated hypoglycaemia; insulin-like growth factor, solitary fibrous tumour, retroperitoneal tumour.

\section{INTRODUCTION}

Spontaneous hypoglycaemia may be caused by insulin secreted from a functional tumour of the pancreatic islet cells, hypoadrenalism, hypopituitarism or liver pathology. Hypoinsulinaemic hypoglycaemia as a paraneoplastic syndrome (non-islet cell tumour hypoglycaemia, $\mathrm{NICTH}$ ) is a rare complication of some tumours ${ }^{[1]}$. Excessive production of insulin-like growth factor-2 (IGF-2) by the tumour cells stimulates insulin receptors and increased glucose use. If this rare syndrome is not suspected, it may be life-threatening and may lead to cerebral hypoxia and death. We report the case of a patient with NICTH caused by a solitary fibrous tumour (SFT). 


\section{CASE REPORT}

A 76-year-old male ex-miner presented to the emergency assessment unit with agitation and confusion and a blood glucose level of 2.1 $\mathrm{mmol} / \mathrm{l}$. There was a history of a weight loss of 5-8 $\mathrm{kg}$ within the previous 6 months. His past medical history included hypertension, benign prostatic hypertrophy, left shoulder hemiarthroplasty, osteoarthritis and age-related macular degeneration. He had no significant family history of illness.

On examination he was afebrile and euvolaemic after the paramedics had resuscitated him with intravenous dextrose. There was an intraabdominal smooth, non-tender, non-ballotable right upper quadrant mass on examination.

During his hospital stay, the patient had multiple episodes of hypoglycaemia that were managed with intravenous dextrose, oral dextrose gel, dexamethasone, corn-based starch in additional to meals, and frequent scheduled snacks as suggested by the dieticians.

The CT scan and abdominal angiogram showed a heterogeneous mass measuring $26 \times 15 \times 18 \mathrm{~cm}$ and with calcification, in the right upper retroperitoneum between the liver and right kidney (Fig. 1). The lesion had a rich blood supply from the branches of the right renal artery and directly from the aorta superior to the right renal artery. There was possible inferior venacaval invasion. There was confluent mediastinal lymphadenopathy of uncertain significance.

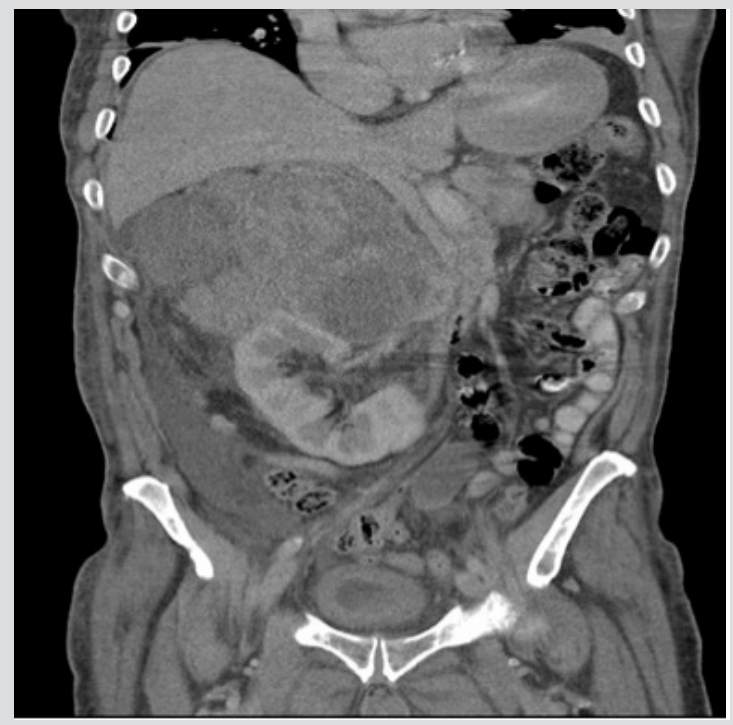

Figure 1: CT scan showing the mass in abdomen.

Histology of the core biopsy showed a monomorphic spindle cell tumour with stromal ropey collagen. The expression of CD34 and BCL2, and lack of other significant markers (CD117, DOG1, AE1/AE3, EMA, S100, Melan A, desmin and CD31) confirmed the diagnosis of SFT. There was no cellular atypia, >3 mitoses per 10 high-power fields (hpf) or necrosis to suggest a definitive diagnosis of a malignant SFT. SFTs are categorised as tumours with intermediate biological potential but where the biological behaviour of individual tumours is notoriously difficult to predict. Tumours $\geq 15 \mathrm{~cm}$ in diameter in patients $\geq 55$ years of age and with $\geq 4$ mitotic figures per $10 \mathrm{hpf}$ have been shown to be associated with a high risk of both metastasis and death ${ }^{[2]}$. With the rich blood supply precluding tumour chemo-embolisation and surgical intervention, the sarcoma multidisciplinary team concluded that the general outcome in this patient was likely to be poor.

Table 1 tabulates the laboratory results when the patient was euglycaemic. The low-normal proinsulin levels exclude the possibility of ectopic insulin secretion, insulinoma or exogenous insulin administration as the cause of hypoglycaemia. The raised IGF-2 levels confirm that this patient had Doege-Potter syndrome ${ }^{[3]}$.

Plasma proinsulin levels, cortisol, IGF-1 and IGF-2 levels were also investigated.

The patient's episodic hypoglycaemia was managed with diet modification including corn-based starch, scheduled snacks and dexamethasone. He responded well to the treatment without further episodes of hypoglycaemia. The patient was stabilised on dexamethasone and discharged with a glucagon pen and counselling to be followed up by the palliative team. 


\begin{tabular}{|l|l|l|}
\hline Test & Result & Reference range \\
\hline Glucose & $8 \mathrm{mmol} / 1$ & $3.0-6.0$ \\
\hline Proinsulin & $3 \mathrm{pmol} / 1$ & $0-10$ \\
\hline Insulin-like growth factor-1 (IGF-1) & $7.7 \mathrm{nmol} / 1$ & $<9$ \\
\hline Insulin-like growth factor-2 (IGF-2) & $123.5 \mathrm{nmol} / 1$ & $<20$ \\
\hline IGF-2:IGF-1 & 16.0 & $<10$ \\
\hline Cortisol & $229 \mathrm{nmol} / 1$ & $140-690$ \\
\hline
\end{tabular}

\section{DISCUSSION}

NICTH linked with excessive production of IGFs and Doege-Potter syndrome ${ }^{[3]}$ was first reported in a patient with a spindle cell tumour with hypoglycaemia of unknown aetiology.

The pathogenesis of NICTH appears to be incompletely processed IGF-2 ('big' IGF). IGF-2 leads to increased skeletal muscle glucose use, suppression of glucagon, growth hormone release and inhibition of hepatic glucose release, with resulting persistent chronic hypoglycaemia. It also has higher affinity for insulin receptors and lower affinity for insulin binding proteins (IGF-BP) ${ }^{[4]}$, mimicking the action of insulin. The IGF-2:IGF-1 ratio of 16 (normal=3:1) in our patient is pathognomonic of NICTH as the cause of hypoglycaemia ${ }^{[4]}$.

The aims of treatment in patients with NICTH include maintaining euglycaemia and where possible, curative excision of the IGF- 2 producing tumour (IGF-2oma). Simple measures such as administration of glucose tablets or liquid, fruit juice or intravenous glucose can be used in the acute setting. The usual medical management for spontaneous hypoglycaemia in instances of insulinomas (which includes diazoxide, octreotide and glucagon) has some limited use in NICTH, but steroids are the mainstay of treatment. Steroids decrease the amount of 'big' IGF-2 by converting pre-pro IGF into IGF-2. They also re-establish the normal IGF:IGF-BP ratio. Supra-physiological doses of recombinant growth hormone increase IGF-BP, preventing interaction with insulin receptors and ultimately decreasing the chances of hypoglycaemia[5]. Definitive treatment depends on the nature of the tumour. If the tumour is resectable, surgery is often curative. The metabolic alterations caused by NICTH are reversible in many patients after complete surgical excision of 'big' IGF-2 producing tumours. A search of the literature from 2004 to 2014 revealed around 58 IGF-2oma case reports, 21 which were SFTs (both malignant and benign). Chemotherapy, embolisation or irradiation may also be considered with a variable success rate in controlling hypoglycaemia.

\section{REFERENCES}

1. Bodnar TW, Acevedo MJ, Pietropaolo M. Management of non-islet-cell tumor hypoglycemia: a clinical review. J Clin Endocrinol Metab $2014 ; 99: 713$.

2. Demicco EG, Park MS, Araujo DM, Fox PS, Bassett RL, Pollock RE, et al. Solitary fibrous tumour: a clinicopathological study of 110 cases and proposed risk assessment model. Mod Pathol 2012;25:1298-1306.

3. Fung EC, Crook MA. Doege-Potter syndrome and 'big-IGF2': a rare cause of hypoglycaemia. Ann Clin Biochem 2011;48:95-96.

4. Teale JD, Wark G. The effectiveness of different treatment options for non-islet cell tumour hypoglycaemia. Clin Endocrinol 2004;60:457-460.

5. Otake S, Kikkawa T, Takizawa M, Oya J, Hanai K, Tanaka N, et al. Hypoglycemia observed on continuous glucose monitoring associated with IGF-2-producing solitary fibrous tumour. J Clin Endocrinol Metab 2015;100:2519-2524. 